\title{
Colonic Mucosa-Associated Lymphoid Tissue Lymphoma
}

\author{
Risaburo Akasaka ${ }^{a}$ Toshimi Chiba $^{a}$ Amit K. Dutta $^{a, c}$ \\ Yosuke Toya $^{\mathrm{a}}$ Tomomi Mizutani $^{\mathrm{a}}$ Tatsuyori Shozushima ${ }^{\mathrm{a}}$ \\ Keinosuke Abe ${ }^{a} \quad$ Masato Kamei $^{a}$ Satoshi Kasugai ${ }^{a}$ \\ Sho Shibata ${ }^{a}$ Yukito Abiko $^{a}$ Naoki Yokoyama ${ }^{a}$ \\ Shuhei Oana $^{a}$ Shigeru Hirota $^{a}$ Masaki Endo $^{a}$ \\ Noriyuki Uesugi ${ }^{b}$ Tamotsu Sugai ${ }^{b}$ Kazuyuki Suzuki ${ }^{a}$ \\ ${ }^{a}$ Division of Gastroenterology and Hepatology, Department of Internal \\ Medicine, and ${ }^{b}$ Division of Molecular Diagnostic Pathology, Department of \\ Pathology, School of Medicine, Iwate Medical University, Morioka, Japan; \\ 'Department of Gastroenterology, Christian Medical College and Hospital, \\ Vellore, India
}

\section{Key Words}

Colonic mucosa-associated lymphoid tissue lymphoma · Polypoid lesions · Colonoscopy

\begin{abstract}
Colonic mucosa-associated lymphoid tissue (MALT) lymphomas are rare and a definitive treatment has not been established. Solitary or multiple, elevated or polypoid lesions are the usual appearances of MALT lymphoma in the colon and sometimes the surface may reveal abnormal vascularity. In this paper we report our experience with four cases of colonic MALT lymphoma and review the relevant literature. The first patient had a smooth elevated lesion in the rectum and histopathologic examination of the biopsy from the lesion showed centrocyte-like cells infiltrating the lamina propria. Endoscopic ultrasonography (EUS) revealed thickening of the submucosa and muscularis propria. The patient underwent radiation therapy, and 9 months later a repeat colonoscopy showed complete resolution of the lesion. In case 2, colonoscopy showed a polyp in the cecum; the biopsy was diagnostic of MALT Iymphoma. EUS detected a hypoechoic lesion confined to the mucosal layer of the colonic wall. The patient underwent endoscopic mucosal resection of the lesion and after 6 years of follow-up there was no evidence of recurrence. The third patient had a sessile elevated lesion in the sigmoid colon for which she underwent sigmoidectomy. Pathological examination of the surgical specimen was suggestive of MALT lymphoma. The last patient had a smooth elevated lesion in the rectum and magnification endoscopy showed irregular vascular pattern. The patient underwent endoscopic submucosal dissection, and biopsy examination showed the tumor to be MALT lymphoma. Although rare, awareness of MALT
\end{abstract}


lymphoma of the colon is important to evaluate the patient appropriately and to plan further management.

\section{Introduction}

Mucosa-associated lymphoid tissue (MALT) is found in various parts of the body, but the predominant site with the highest volume of this tissue is the gastrointestinal tract. It plays an important role in immune surveillance, mucosal regeneration and is also involved in carcinogenesis [1]. In the colon, lymphoid tissue occurs mainly as isolated lymphoid follicles which are composed mainly of B lymphocytes [1]. The cells in MALT may occasionally undergo abnormal proliferation and give rise to lymphoma of the MALT type [2]. The term MALT lymphoma was first described by Isaacson and Wright in 1983 [3]. The World Health Organization (WHO) classification categorizes it as extra-nodal marginal zone B cell lymphoma of the MALT type. This type of lymphoma mainly occurs in the stomach and small intestine and rarely in the colon [4]. Among the lymphomas seen in the colon, the commonest type is diffuse large B cell lymphoma, and MALT type lymphoma accounts for less than $20 \%$ cases [5]. Due to infrequent occurrence, there are only few reports of colonic MALT type lymphoma, and absence of sufficient data makes evaluation and management of these patients challenging [6]. Here we describe four patients with colonic MALT lymphoma, each with a different presentation and management strategy. This highlights the variability in the presentation of this condition and the need for an individualized management strategy in the absence of a common consensus on management.

\section{Case 1}

A 57-year-old female patient presented with a history of bleeding per rectum for the past month in 2006. There was no history of weight loss, fever or any other significant symptom. Blood evaluation revealed hemoglobin $11.6 \mathrm{~g} / \mathrm{dl}$, total protein $7.5 \mathrm{~g} / \mathrm{dl}$, serum albumin $4.7 \mathrm{~g} / \mathrm{dl}$, total leucocyte count $3,310 / \mu \mathrm{l}$, lactate dehydrogenase (LDH) $156 \mathrm{U} / \mathrm{l}$ and CA19-9 7.4 U/ml. Colonoscopy done during the evaluation of the source of gastrointestinal blood loss showed a smooth elevated lesion $>3 \mathrm{~cm}$ in diameter in the rectum (fig. 1a). Pathological findings of the biopsy sample from the rectal lesion showed infiltrated centrocyte-like (CCL) cells infiltrating the lamina propria (fig. 1b). Endoscopic ultrasonography (EUS) indicated thickening of the submucosa and muscularis propria. Contrastenhanced computed tomography (CECT) of the abdomen showed thickening of the rectal wall, and there was no evidence of lesion or lymphadenopathy at any other site. Whole-body gallium scintigraphy was normal. A diagnosis of MALT lymphoma of the colon was made and the patient received $30 \mathrm{~Gy}$ of radiation therapy localized to the rectal lesion. Nine months after radiation therapy, a follow-up colonoscopy showed complete resolution of the elevated lesion (fig. 1c).

\section{Case 2}

The second patient was a 64-year-old lady who was seen at our hospital for the evaluation of chronic constipation in 2006. She had no history of gastrointestinal bleeding, anorexia, weight loss or fever. Blood evaluation showed hemoglobin $11.8 \mathrm{~g} / \mathrm{dl}$, total protein $6.9 \mathrm{~g} / \mathrm{dl}$, serum albumin $4.1 \mathrm{~g} / \mathrm{dl}$, total leucocyte count 3,980/ $\mu \mathrm{l}$, LDH $240 \mathrm{U} / \mathrm{l}$ and CA19-9 $11.7 \mathrm{U} / \mathrm{ml}$. Colonoscopy performed for evaluation of constipation detected a polyp in the cecum ( fig. 2a); the biopsy was suggestive of MALT lymphoma. EUS was done to assess the depth of the lesion to plan therapy. It showed a hypoechoic lesion which was confined to the mucosal layer of the cecum, and endoscopic mucosal resection was 
considered appropriate for therapy. Pathological findings in the endoscopically resected specimen showed atypical lymphocytes with CCL cells in the mucosal layer, and immunohistochemical stains for L26 and CD79a were positive (fig. 2b, c) which confirmed the diagnosis of MALT lymphoma. The patient is on regular follow-up and the last colonoscopy done in early 2012 showed no evidence of recurrent lesion.

\section{Case 3}

A 50-year-old female was detected to have a positive fecal occult blood test which was performed to screen for colorectal malignancy at another hospital. She was referred to our hospital for further detailed evaluation. She had no abdominal or extra-abdominal symptoms such as altered bowel habit, anorexia or weight loss. Blood evaluation revealed hemoglobin $12.7 \mathrm{~g} / \mathrm{dl}$, total protein $6.9 \mathrm{~g} / \mathrm{dl}$, serum albumin $4.0 \mathrm{~g} / \mathrm{dl}$, total leucocyte count 4,500/ $\mu \mathrm{l}$ and LDH $318 \mathrm{U} / \mathrm{l}$. Colonoscopy done during the course of evaluation revealed a sessile elevated lesion with angiectasia on the surface in the sigmoid colon (fig. $3 \mathrm{a}$ ). The biopsy was suggestive of MALT lymphoma. CECT of the abdomen did not show lymphadenopathy or lesion at any other site. EUS showed the lesion to extend to the submucosal layer and surgical resection was planned. The patient underwent sigmoidectomy which revealed an $18 \mathrm{~mm}$ tumor (fig. 3b). Pathological findings showed diffuse atypical lymphocytes with CCL cells in the mucosal and submucosal layers. Immunohistochemical staining for $\mathrm{Bcl}-2$ was positive and was negative for CD5, CD10 and cyclin D1 (fig. 3c, d). A final diagnosis of colonic MALT lymphoma was made.

\section{Case 4}

The last patient was a 57-year-old lady who presented to us for the evaluation of lower abdominal pain. The origin of pain was unclear and she had no other significant symptoms. Blood investigations were within normal limits. CECT of the abdomen did not show any abnormality which could explain her symptoms. In view of localization of pain in the lower abdomen, colonoscopy was performed which showed a smooth elevated lesion in the rectum. Detailed assessment of the rectal lesion with magnification endoscopy showed an irregular vascular pattern on its surface ( $\underline{\text { fig. } 4 \mathrm{a}}$ ). The patient underwent endoscopic submucosal dissection for removal of the lesion. Histopathologic findings showed diffuse atypical lymphocytes with CCL cells in the mucosal and submucosal layers (fig. 4b, c) which supported the diagnosis of MALT lymphoma.

\section{Discussion}

Among the four cases presented here, only one (case 1) had symptoms directly related to the lesion and the rest were incidentally detected during evaluation of unrelated symptoms. The rectosigmoid region was the commonest site of the lesions and all our patients were females. Diagnostic and management strategy depended on the size, vascularity and depth of the lesions, and two patients underwent successful endoscopic therapy with complete resection of the lesions. For larger and deeper lesions surgery or radiation therapy were considered.

Colorectal lymphomas comprise about $10-20 \%$ of gastrointestinal lymphomas and account for $<1 \%$ of large bowel malignancies [7]. Our case series highlights the variability in the presentation of this condition. It also emphasizes the need for an individualized management strategy in the absence of a common consensus on treatment of this rare entity. The current knowledge on its diagnosis and management is briefly presented here. Before invasive evaluation, baseline blood tests including 
blood counts, total protein and serum albumin should be performed to assess the general condition of the patient.

\section{Endoscopy}

Colonoscopy is critical in the detection and characterization of the lesion as well as for obtaining biopsies. Colonoscopic findings in patients with MALT lymphoma are categorized as follows: solitary protrusion, multiple protrusions and numerous small polyps. Solitary or multiple protrusions are the most common pattern of MALT lymphoma in the colon. A nodular surface, vascular ectasia and ulceration may be typical features of larger lesions, and the abnormal vascular pattern may be easily detected by using narrow-band imaging $[8,9]$.

\section{Diagnostic Confirmation and Assessing the Extent of the Lesion}

Establishing the diagnosis and extent of the disease facilitates the planning of appropriate treatment. However, biopsy may not always be feasible due to the increased vascularity seen with some lesions. Histological features of MALT lymphoma include infiltration by CCL cells. Immunophenotyping is very useful in confirming the diagnosis. Immunohistochemical analyses can distinguish MALT lymphoma from other low-grade lymphomas by detecting positivity for superficial immunoglobulins and pan B antigens - CD19, CD20, CD79 without expression of CD5, CD10, CD23 and cyclin D1 $[10,11]$. Tumor cells are also positive for Bcl-2 and negative for intracytoplasmic immunoglobulins, UCHL-1/CD45R0 and p53 [11]. Local staging (tumour depth) can be done using EUS while assessment of lymphadenopathy and other distant lesions requires modalities like CECT of the abdomen. The Lugano modification of the Ann Arbor classification is used for staging, and this may necessitate the performance of other tests such as chest CECT and bone marrow examination [12].

\section{Treatment}

The treatment of gastric MALT lymphoma is well established, whereas the treatment of colonic lymphoma is less clear. The role of Helicobacter pylori and its response to antibiotics makes the treatment of gastric lymphoma very effective. Colorectal MALT lymphomas do not have the same association with $H$. pylori infection and are treated as a different clinical entity [13]. In the absence of standardized treatment, various treatment methods have been used which include surgery, chemotherapy, radiation and endoscopic resection [6]. As a causative organism has not been identified, the role of antibiotics is controversial [6]. Thus, because of divergent therapies reported in various studies, there are as yet no clear therapeutic guidelines for treatment of MALT lymphoma of the colon [14]. Surgical resection may be effective when a colorectal MALT lymphoma does not respond to eradication therapy or chemotherapy and is localized without dissemination [15].

\section{Conclusion}

MALT lymphoma of colon is a rare but clinically important condition which requires proper evaluation for guiding therapy. More data are required to provide better insight into this disease and to aid development of uniform evaluation and treatment strategies. 


\begin{tabular}{r|l|l|l}
$\begin{array}{r}\text { Case Reports in } \\
\text { Gastroenterology }\end{array}$ & $\begin{array}{l}\text { Case Rep Gastroenterol 2012;6:569-575 } \\
\text { DOI: 10.1159/000342726 }\end{array}$ & $\begin{array}{l}\text { Published online: } \\
\text { August 29, 2012 }\end{array}$ & $\begin{array}{l}\text { @ 2012 S. Karger AG, Basel } \\
\text { ISSN 1662-0631 } \\
\text { www.karger.com/crg }\end{array}$ \\
\hline
\end{tabular}
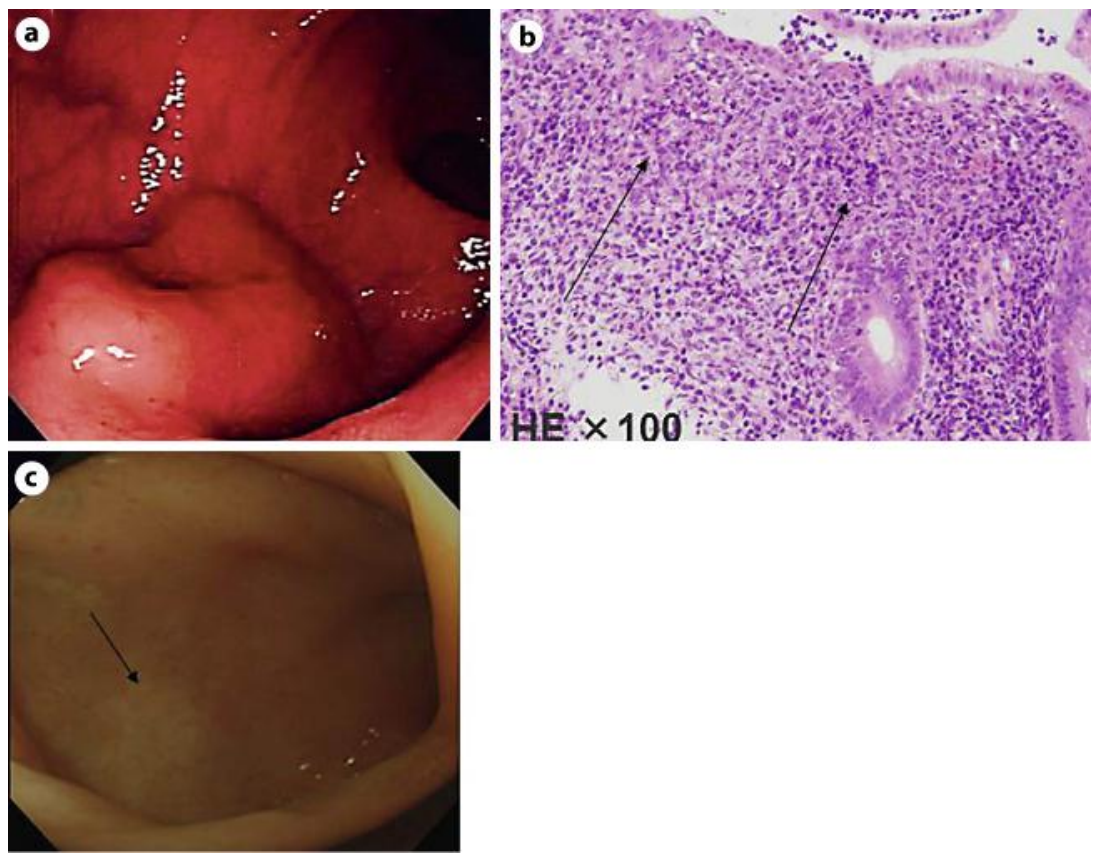

Fig. 1. Case 1. a Colonoscopy showing a smooth elevated lesion $>3 \mathrm{~cm}$ in diameter in the rectum. b Pathological findings of the biopsy sample from the rectal lesion showing infiltrated CCL cells in the lamina propria. c Follow-up colonoscopy 9 months after radiation therapy showing resolution of the elevated lesion in the rectum.
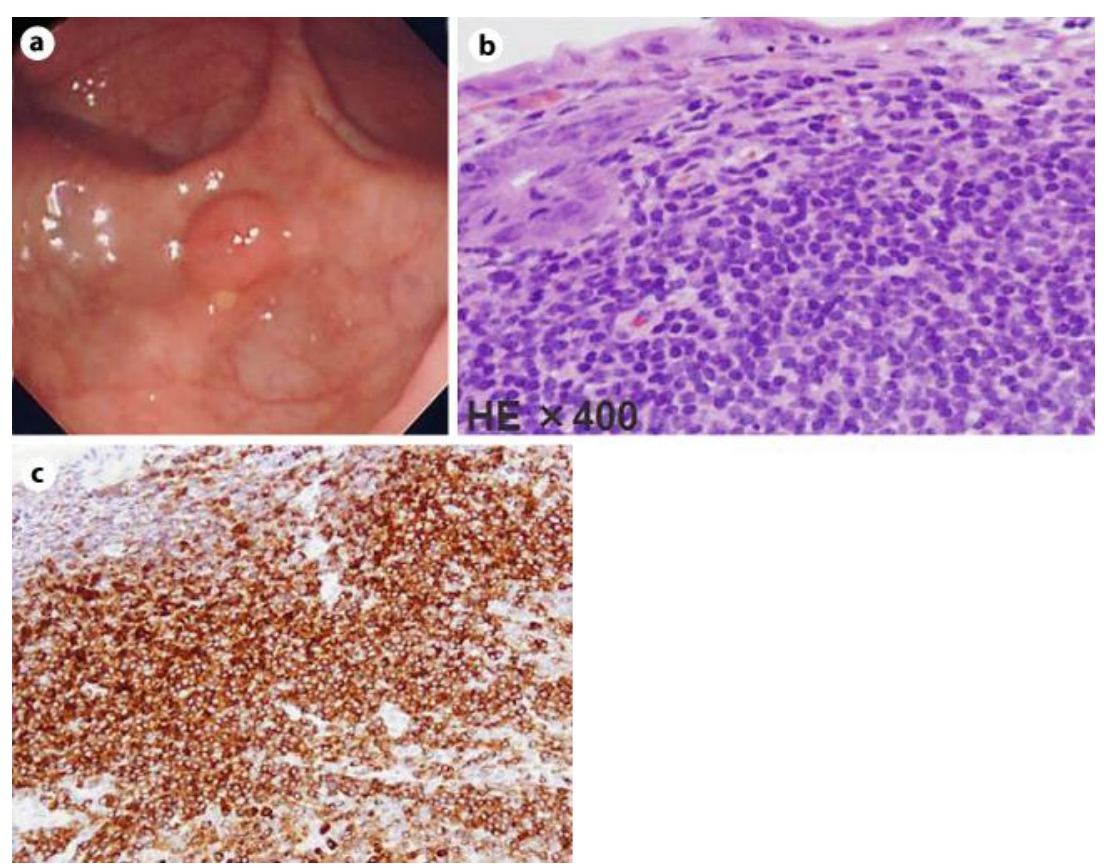

Fig. 2. Case 2. a Colonoscopy showing a polyp in the cecum. b, c Pathological findings in the endoscopically resected specimen showing atypical lymphocytes with CCL cells in the mucosal layer; the immunohistochemical stain for CD79a was positive. 

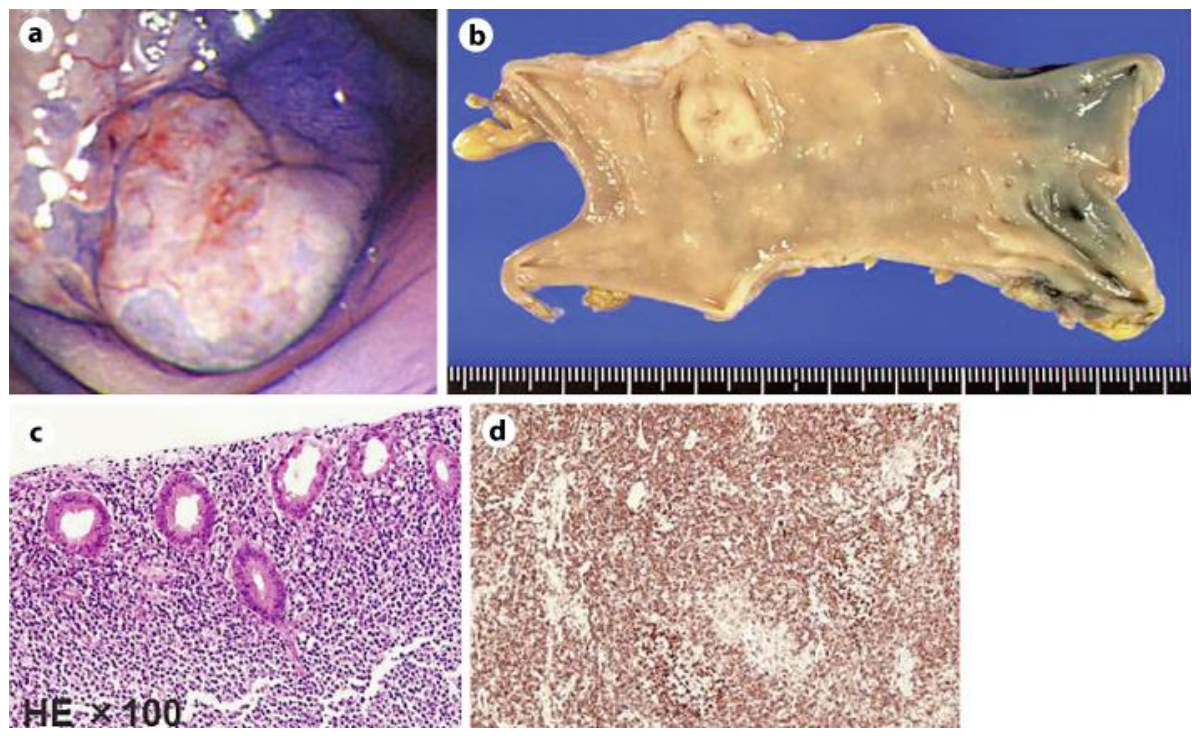

Fig. 3. Case 3. a Colonoscopy showing a sessile elevated lesion with angiectasia in the sigmoid colon. b Sigmoidectomy specimen showing an $18 \mathrm{~mm}$ tumor. c, d Pathological findings in the resected specimen showing diffuse atypical lymphocytes with CCL cells in the mucosa and submucosal layer; the immunohistochemical stain for Bcl-2 was positive.

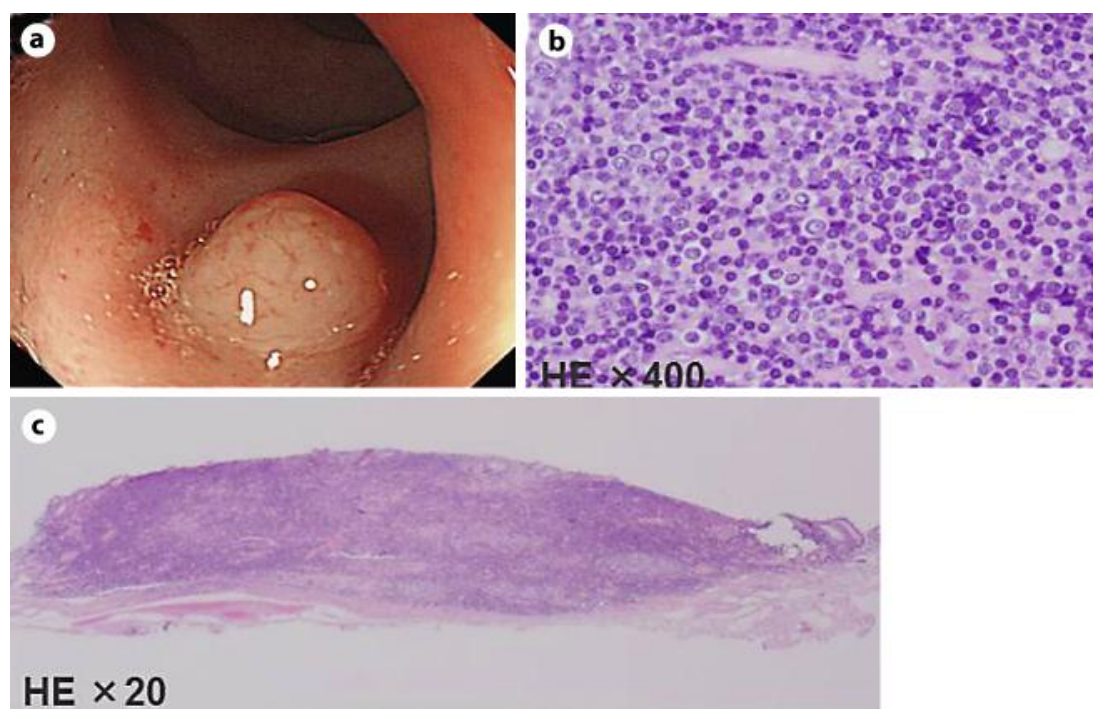

Fig. 4. Case 4. a Colonoscopy with magnification showing a smooth elevated lesion with irregularity of the vascular pattern in the rectum. b, $\mathbf{c}$ Pathological findings in the endoscopically resected specimen showing diffuse atypical lymphocytes with CCL cells in the mucosa and submucosal layer. 


\section{References}

1 Sipos F, Muzes G: Isolated lymphoid follicles in colon: switch points between inflammation and colorectal cancer? World J Gastroenterol 2011;17:1666-1673.

-2 Thieblemont C, Bastion Y, Berger F, et al: Mucosa-associated lymphoid tissue gastrointestinal and nongastrointestinal lymphoma behavior: analysis of 108 patients. J Clin Oncol 1997;15:1624-1630.

-3 Isaacson P, Wright DH: Malignant lymphoma of mucosa-associated lymphoid tissue. A distinctive type of B-cell lymphoma. Cancer 1983;52:1410-1416.

-4 Koch P, del Valle F, Berdel WE, et al: Primary gastrointestinal non-Hodgkin's lymphoma: I. Anatomic and histologic distribution, clinical features, and survival data of 371 patients registered in the German Multicenter Study GIT NHL 01/92. J Clin Oncol 2001;19:3861-3873.

$>5$ Howell JM, Auer-Grzesiak I, Zhang J, et al: Increasing incidence rates, distribution and histological characteristics of primary gastrointestinal non-Hodgkin lymphoma in a North American population. Can J Gastroenterol 2012;26:452-456.

-6 Ahlawat S, Kanber Y, Charabaty-Pishvaian A, et al: Primary mucosa-associated lymphoid tissue (MALT) lymphoma occurring in the rectum: a case report and review of the literature. South Med J 2006;99: 1378-1384.

7 Wong MT, Eu KW: Primary colorectal lymphomas. Colorectal Dis 2006;8:586-591.

-8 Saito T, Toyoda H, Yamaguchi M, et al: Ileocolonic lymphomas: a series of 16 cases. Endoscopy 2005;37: 466-469.

-9 Cyrany J, Pinter M, Tycova V, et al: Trimodality imaging of colonic lymphoma. End oscopy 2009;41 (suppl 2):E1-E2.

10 Toyomasu Y, Tsutsumi S, Yamaguchi S, Mochiki E, Asao T, Kuwano H: Laparoscopy-assisted ileocecal resection for mucosa-associated lymphoid tissue lymphoma of the appendix: case report. Hepatogastroenterology 2009;56:1078-1081.

11 Shimura T, Kuwano H, Kashiwabara K, et al: Mucosa-associated lymphoid tissue lymphoma of the extrahepatic bile duct. Hepatogastroenterology 2005;52:360-362.

$\checkmark 12$ Rohatiner A, d'Amore F, Coiffier B, et al: Report on a workshop convened to discuss the pathological and staging classifications of gastrointestinal tract lymphoma. Ann Oncol 1994;5:397-400.

13 Quayle FJ, Lowney JK: Colorectal lymphoma. Clin Colon Rectal Surg 2006;19:49-53.

$\checkmark 14$ Li B, Shi YK, He XH, et al: Primary non-Hodgkin lymphomas in the small and large intestine: clinicopathological characteristics and management of 40 patients. Int J Hematol 2008;87:375-381.

15 Matsuo S, Mizuta Y, Hayashi T, et al: Mucosa-associated lymphoid tissue lymphoma of the transverse colon: a case report. World J Gastroenterol 2006;12:5573-5576. 